\title{
Emission spectra of Cs-He excimers in cold helium gas
}

AUTHOR(S):

Enomoto, K; Hirano, K; Kumakura, M; Takahashi, Y; Yabuzaki, T

\section{CITATION:}

Enomoto, K ...[et al]. Emission spectra of Cs-He excimers in cold helium gas. PHYSICAL REVIEW A 2002, 66(4): 042505.

ISSUE DATE:

2002-10

URL:

http://hdl.handle.net/2433/49830

RIGHT:

Copyright 2002 American Physical Society 
PHYSICAL REVIEW A 66, 042505 (2002)

\title{
Emission spectra of Cs-He excimers in cold helium gas
}

\author{
K. Enomoto, ${ }^{*}$ K. Hirano, M. Kumakura, Y. Takahashi, and T. Yabuzaki \\ Department of Physics, Graduate School of Science, Kyoto University, Kyoto 606-8502, Japan
}

(Received 27 April 2002; published 15 October 2002)

\begin{abstract}
We observed broadband emission spectra of Cs-He excimers $\left(\mathrm{Cs}^{*} \mathrm{He}\right)$ in cold $\mathrm{He}$ gas $(1.3-100 \mathrm{~K})$, when $\mathrm{Cs}$ atoms were excited to the $6{ }^{2} \mathrm{P}$ states. We calculated the emission spectrum from each vibrational state of Cs*He using Pascale's semiempirical Cs-He potential energy curves [J. Pascale, Phys. Rev. A 28, 632 (1983)], and compared the results with the observed spectra. From the measured spectral shape, we estimated the relative populations of the vibrational states of $\mathrm{Cs}^{*} \mathrm{He}$ at various $\mathrm{He}$ gas densities, and estimated the predissociation rate of $\mathrm{Cs} * \mathrm{He}$ in the $A^{2} \Pi_{1 / 2}$ state. Moreover, a broadband emission spectrum of $\mathrm{Cs}^{*} \mathrm{He}_{2}$ was also observed, which was compared with that observed in liquid He.
\end{abstract}

DOI: 10.1103/PhysRevA.66.042505

\section{INTRODUCTION}

Alkali-metal atoms in gaseous He have been investigated by spectroscopic methods for a long time. The presence of He causes pressure broadening of resonance lines of alkalimetal atoms. The wide red wings of the $D$ emission lines of alkali-metal atoms have been investigated extensively [1-6], and recently precise measurements of the pressure broadening of the $\mathrm{Rb} D$ absorption lines were reported by Romalis et al. [7]. These resonance line profiles were utilized to evaluate the alkali-metal-He interatomic potentials, which are difficult to determine precisely because of the weakness of the attractive interaction. However, clear observation of fluorescence from alkali-metal-He excimers and exciplexes, which leads to more precise evaluation of the interatomic potentials, has not occurred until recently. This is mainly because most of the experiments were carried out at or above room temperature, so that the thermal energy exceeded the small binding energy of alkali-metal and He atoms [for example, the potential well depth for the first excited state $\left(A^{2} \Pi\right)$ is calculated to be $112 \mathrm{~cm}^{-1}$ for Cs-He [8]].

In the last decade, spectroscopic studies of alkali-metal atoms in cold $\mathrm{He}$ environments have made great progress [9], and exciplex formation in (or on) condensed He has been discussed to explain the unique features of the fluorescence. For example, in superfluid bulk liquid $\mathrm{He}$, alkali-metal- $\mathrm{He}_{n}$ exciplex formation explained the absence of fluorescence from light alkali-metal atoms $(\mathrm{Li}, \mathrm{Na})$ [10-12], the absence of the $D_{2}$ line emission from excited $\mathrm{Rb}$ and Cs atoms [13], and the quenching of the $\mathrm{Rb} 5{ }^{2} P_{1 / 2}$ state in pressurized liquid $\mathrm{He}$ [14]. Also, in solid $\mathrm{He}, \mathrm{Rb}$ atoms do not emit visible fluorescence whereas Cs atoms do. Therefore, light absorption was used to detect nonfluorescing $\mathrm{Rb}$ atoms in a recent optical-rf double resonance experiment [15]. These interesting qualitative differences between different alkali-metal atoms can be explained in the context of the difference in spin-orbit coupling strength of the $P$ state [12]. In liquid He, we observed infrared emission when heavy alkali-metal atoms (K,Rb,Cs) were excited to the first $P$ state [16]. The emission is expected to be from the largest alkali-metal$\mathrm{He}_{n_{\max }}$ exciplexes, where $n_{\max }$ represents the maximum

*Electronic address: enomoto@yagura.scphys.kyoto-u.ac.jp
PACS number(s): 33.20.Ea, 34.30.+h, 67.40.Yv

number of He atoms bonding to an alkali-metal atom. The exciplex formation in liquid $\mathrm{He}$ is so rapid that transient exciplexes, such as $\mathrm{Cs}^{*} \mathrm{He}$, have not been observed. Thus more dilute $\mathrm{He}$ environments are required for investigation of transient small exciplexes, to obtain information on the alkali-metal-He interatomic potentials and on the maximum number $n_{\text {max }}$. Recently, fluorescence from $\mathrm{K}^{*} \mathrm{He}_{n}, \mathrm{Na}^{*} \mathrm{He}_{n}$ $(n=1,2)$ [17], and $\mathrm{Rb}^{*} \mathrm{He}$ [18] was observed in such an environment. These exciplexes were produced by photodetachment from the surface of He nanodroplets. In these studies the observed spectra were compared with calculated spectra using Pascale's alkali-metal-He potentials [8]. Time resolved studies have also been reported [19,20], and Schulz et al. suggested that $n_{\max }$ for $\mathrm{K}-\mathrm{He}$ exciplexes is 4 , by observing the mass spectrum of exciplexes [20]. However, $\mathrm{Cs}^{*} \mathrm{He}$ has not been observed yet in spite of the interesting feature that it has a large difference from other alkali-metal atoms in the spin-orbit coupling strength. Thus we chose Cs, and we also intend to determine $n_{\max }$ for Cs-He exciplexes.

In the present work we have used the simplest environment of dilute He: cold He gas. This environment has an advantage over He nanodroplets, that is, we can change the temperature and the density of $\mathrm{He}$ gas over wide ranges. So far such a cold He gas has been used by Jakubek et al. for observing the fluorescence from $\mathrm{Ag}^{*} \mathrm{He}_{n}(n=1,2)[21]$.

In Sec. II we describe the experimental setup, and in Sec. III we show observed emission spectra of $\mathrm{Cs}^{*} \mathrm{He}_{n}$ ( $n$ $=1,2$ ). In Sec. IV we compare the measured spectra of $\mathrm{Cs}^{*} \mathrm{He}$ with theoretical ones calculated from the Cs-He potential curves reported by Pascale [8], and discuss the predissociation rate of $\mathrm{Cs}^{*} \mathrm{He}$ in the $A^{2} \Pi_{1 / 2}$ state. We also show an emission spectrum of $\mathrm{Cs}$ in liquid $\mathrm{He}$.

\section{EXPERIMENTAL SETUP}

The experimental setup is illustrated in Fig. 1. The experiment was performed with a Pyrex glass cell $(2 \mathrm{~cm} \times 2 \mathrm{~cm}$ $\times 3 \mathrm{~cm}$ rectangular solid) containing a small amount of Cs metal and $\mathrm{He}$ gas at a pressure of about $3.5 \mathrm{~atm}$ at room temperature (the density was about $9 \times 10^{19} \mathrm{~cm}^{-3}$ ). After the glass cell was evacuated to $\sim 10^{-6}$ torr and baked at $500 \mathrm{~K}$ for about $20 \mathrm{~h}$, Cs metal was transferred by distillation. To fill the cell with $\mathrm{He}$ gas at a high density, we immersed the 


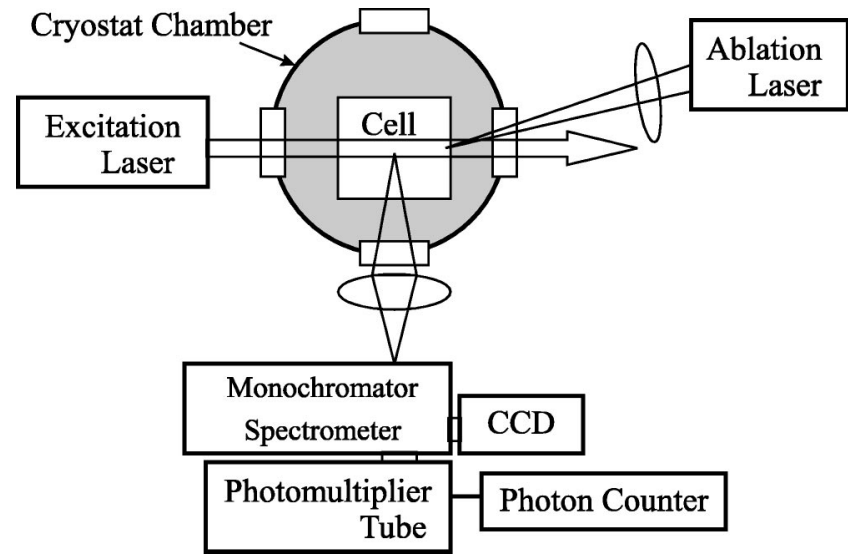

FIG. 1. Experimental setup.

cell in liquid nitrogen $(77 \mathrm{~K})$, introduced the He gas under 1 atm, and then sealed it off.

The cell was immersed in superfluid liquid He for cell temperatures $T<2.17 \mathrm{~K}$ and it was placed in cold gaseous He for $T>2.17 \mathrm{~K}$, in a cryostat with optical windows. Below $1.9 \mathrm{~K}$ the $\mathrm{He}$ gas in the cell liquified. The $\mathrm{He}$ gas density was estimated from the saturated vapor pressure at a given temperature. Details of this cryostat chamber and the method of preparing cells have been described in Ref. [22].

To produce Cs vapor in the cold cell we adopted the laser ablation method; the second harmonic of pulsed $\mathrm{Nd}: \mathrm{YLiF}_{4}$ laser radiation (pulse energy $100 \mu \mathrm{J}$, repetition rate $1 \mathrm{kHz}$, wavelength $523 \mathrm{~nm}$ ) was focused on a small area of the cell wall, which was almost transparent but certainly covered with a thin film or small particles of Cs. After a few second irradiation, we obtained cold atomic Cs gas with a density of $10^{8}-10^{10} \mathrm{~cm}^{-3}$. The increase of the cell temperature caused by the laser irradiation was measured to be less than $0.01 \mathrm{~K}$ for $T<1.9 \mathrm{~K}$ and less than $0.5 \mathrm{~K}$ for $T>1.9 \mathrm{~K}$. This temperature increase was confirmed by observing the small broadening of the $D_{2}\left(6{ }^{2} S_{1 / 2} \rightarrow 6{ }^{2} P_{3 / 2}\right)$ excitation line. With respect to the preparation of cold alkali-metal atomic gas we previously reported the light-induced atom desorption (LIAD) method, which is a very effective way to produce cold gaseous atoms for $\mathrm{Rb}$ and $\mathrm{K}$ [22]. However, we did not adopt the LIAD method in the present work, because it is not efficient above the superfluid temperature and does not work effectively for Cs.

The gaseous Cs atoms produced were excited by a cw diode laser beam (typical intensity $1 \mathrm{~mW} / \mathrm{mm}^{2}$ ), which was tuned to the $D_{1}$ line $\left(6^{2} S_{1 / 2} \rightarrow 6^{2} P_{1 / 2}\right)$ at $11181 \mathrm{~cm}^{-1}$ $(894.3 \mathrm{~nm})$ or the $D_{2}$ line $\left(6^{2} S_{1 / 2} \rightarrow 6^{2} P_{3 / 2}\right)$ at $11736 \mathrm{~cm}^{-1}$ $(852.1 \mathrm{~nm})$. The fluorescence from a small volume in the excitation beam was introduced into a spectrometer and detected with a liquid- $\mathrm{N}_{2}$-cooled charge coupled device $(\mathrm{CCD})$ (Princeton Instruments, LN/CCD-1100PF). The resolution of the spectrometer-CCD system was about $16 \mathrm{~cm}^{-1}$ and the available spectral range was from the visible range to about $10000 \mathrm{~cm}^{-1}$. For the longer wavelength region (wave number down to $6250 \mathrm{~cm}^{-1}$ ), we used an ir-sensitive photomultiplier tube (Hamamatsu, R5509-71) connected to a monochromator, with a resolution of about $60 \mathrm{~cm}^{-1}$. The

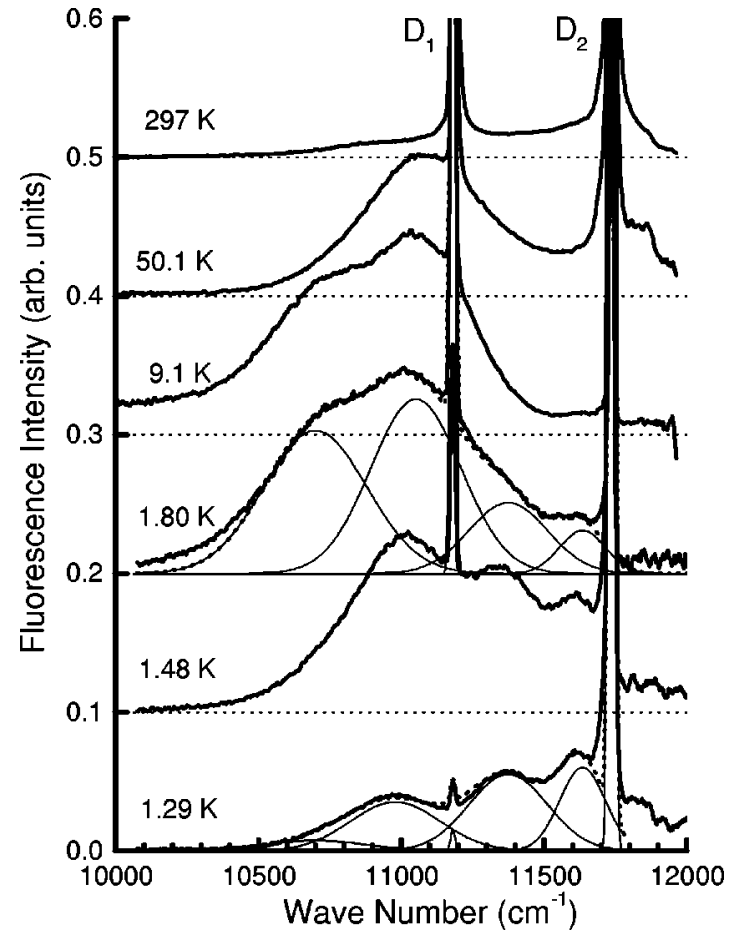

FIG. 2. Emission spectra in the case of $D_{2}$ excitation at various temperatures (thick lines). The superpositions (dotted lines) of six Gaussian curves (thin lines) are fitted to the observed spectra at 1.29 $\mathrm{K}$ and $1.80 \mathrm{~K}$. Above $1.9 \mathrm{~K}$ the $\mathrm{He}$ gas density was 9 $\times 10^{19} \mathrm{~cm}^{-3}$, and below $1.9 \mathrm{~K}$ it was given by the saturated vapor pressure: $6.7 \times 10^{19} \mathrm{~cm}^{-3}$ at $1.80 \mathrm{~K}, 2.1 \times 10^{19} \mathrm{~cm}^{-3}$ at $1.48 \mathrm{~K}$, and $8.4 \times 10^{18} \mathrm{~cm}^{-3}$ at $1.29 \mathrm{~K}$.

sensitivities of both detection systems were calibrated by using a commercial standard lamp.

To observe the emission spectrum in liquid $\mathrm{He}$, we immersed bulk Cs metal in liquid $\mathrm{He}$ at $1.6 \mathrm{~K}$, and sputtered its surface with the pulsed $\mathrm{Nd}: \mathrm{YLiF}_{4}$ laser $(100 \mu \mathrm{J} /$ pulse, 523 $\mathrm{nm})$. Following a dark period of $50 \mu \mathrm{s}$ after each sputtering pulse, a chopped cw Ti: $\mathrm{Al}_{2} \mathrm{O}_{3}$ laser excited the produced $\mathrm{Cs}$ atoms through the $D_{2}$ line [intensity $\sim 300 \mathrm{~mW} / \mathrm{mm}^{2}$, wave number $\left.12034 \mathrm{~cm}^{-1}(831.0 \mathrm{~nm})\right]$.

\section{OBSERVED EMISSION SPECTRA}

The laser-induced fluorescence spectra observed through $D_{2}$ excitation $\left(11736 \mathrm{~cm}^{-1}\right)$ at various temperatures are shown in Fig. 2. The spectra shown have been normalized by the total fluorescence intensity integrated over the observed wave number range. As reported in Ref. [1], at room temperature we observed only the $D$ lines of atomic Cs with wide wings. However, at low temperatures $(T<100 \mathrm{~K})$, a broad spectrum ranging from the $D_{2}$ line to about $10300 \mathrm{~cm}^{-1}$ was clearly observed. This is the spectrum of $\mathrm{Cs}^{*} \mathrm{He}$ excimers as discussed in Sec. IV C. A weak red wing around $10000 \mathrm{~cm}^{-1}$ observed at $3-15 \mathrm{~K}$ is from $\mathrm{Cs}^{*} \mathrm{He}_{2}$ as discussed later.

The observed spectral profile was found to depend strongly on the temperature $T$ below $1.9 \mathrm{~K}$, where the gaseous He density in the cell changed drastically with $T$. The 


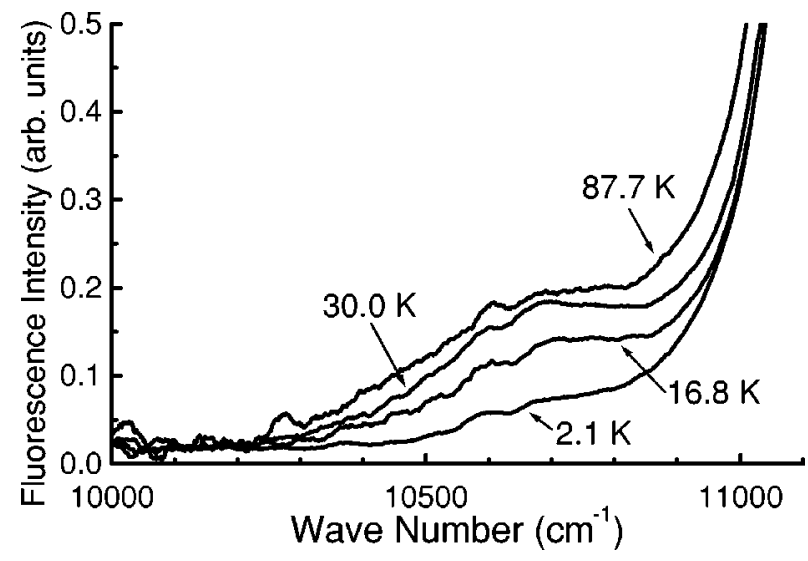

FIG. 3. Emission spectra in the case of $D_{1}$ excitation at various temperatures. They are normalized by the peak intensity of the $D_{1}$ emission line.

observed spectra were composed of four broad components and two atomic $D_{1}$ and $D_{2}$ lines, so that we fitted the superposition of six Gaussian curves to the observed spectra, as shown in Fig. 2. We found that the center wave numbers of the four broad Gaussian curves were about $10695 \mathrm{~cm}^{-1}$, $11070 \mathrm{~cm}^{-1}, 11380 \mathrm{~cm}^{-1}$, and $11635 \mathrm{~cm}^{-1}$, and the full widths at half maximum (FWHMs) were, respectively, about $440 \mathrm{~cm}^{-1}, 390 \mathrm{~cm}^{-1}, 295 \mathrm{~cm}^{-1}$, and $190 \mathrm{~cm}^{-1}$. Comparing these four best-fitted Gaussians at the temperatures 1.80 $\mathrm{K}$ and $1.29 \mathrm{~K}$, we found that the positions and widths of the four components did not change, in spite of large changes of the spectral profile, except for a slight red shift $\left(-80 \mathrm{~cm}^{-1}\right)$ of the component at $11070 \mathrm{~cm}^{-1}$ with decreasing $T$. These broad components correspond to the emission spectra from the vibrational states of $\mathrm{Cs}^{*} \mathrm{He}$, as discussed in Sec. IV C. At about $1.9 \mathrm{~K}$ the components at $10695 \mathrm{~cm}^{-1}$ and $11070 \mathrm{~cm}^{-1}$ were dominant, and at about $1.3 \mathrm{~K}$ the former component became very weak. As the temperature increased from $1.9 \mathrm{~K}$, the former component decreased fast and the latter decreased slowly. The ratio of the total intensity of the emission from $\mathrm{Cs}^{*} \mathrm{He}$ to that of the $D$ lines from Cs gradually decreased, for example, it was 3.8 at $2.1 \mathrm{~K}$ and 0.7 at $50 \mathrm{~K}$, although the $\mathrm{He}$ gas density was constant $\left(9 \times 10^{19} \mathrm{~cm}^{-3}\right)$.

We observed also the fluorescence induced by $D_{1}$ excitation $\left(11181 \mathrm{~cm}^{-1}\right)$. In this case a very weak broad component was observed on the red wing of the $D_{1}$ line at temperatures above $10 \mathrm{~K}$ (Fig. 3). No other emission line was observed. At temperatures below $10 \mathrm{~K}$ the emission spectra were almost the same as that at $2.1 \mathrm{~K}$. With increasing $T$, the intensity of the broad component became larger, and the fraction of the broadband emission in the total emission intensity had the maximum value $\sim 0.01$ at $35 \mathrm{~K}$. For $T>35 \mathrm{~K}$ it gradually merged into the wing of the $D_{1}$ line. This component had almost the same position and width as those of the spectral component at $10695 \mathrm{~cm}^{-1}$ observed in the $D_{2}$ excitation case. This is the emission from $\mathrm{Cs} * \mathrm{He}$ in the $A^{2} \Pi_{1 / 2}$ state, as discussed in Sec. IV B.

We also paid attention to the red wing of the $\mathrm{Cs}^{*} \mathrm{He}$ spectra in the range of $9500-10500 \mathrm{~cm}^{-1}$ observed in the case of $\mathrm{D}_{2}$ excitation. Figure 4 shows the red wing at $2.1 \mathrm{~K}$ and 6.6

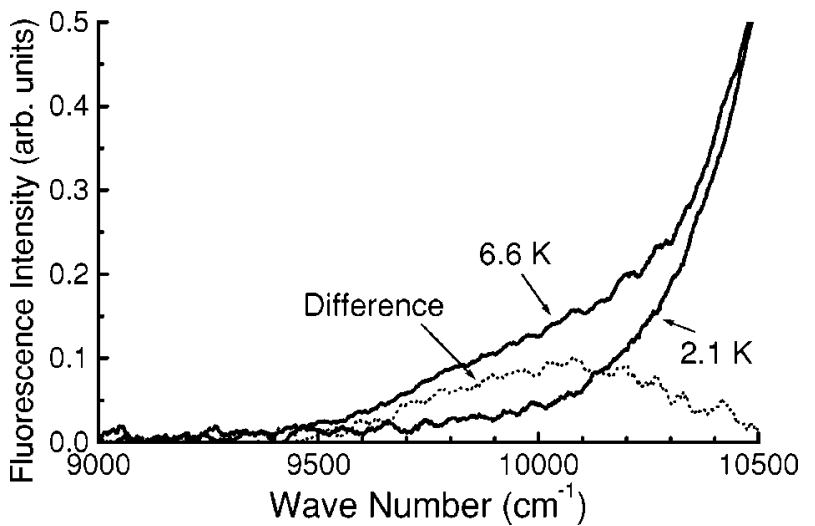

FIG. 4. Emission spectra in an infrared region at $6.6 \mathrm{~K}$ and 2.1 $\mathrm{K}$ (solid lines) in the case of $D_{2}$ excitation. They are normalized by the intensity of the spectral component at $10695 \mathrm{~cm}^{-1}$. The dotted line shows the difference between these two spectra.

$\mathrm{K}$, where we see the existence of a weak spectral component around $10000 \mathrm{~cm}^{-1}$ at $6.6 \mathrm{~K}$. This line was observed only in the temperature range from $3 \mathrm{~K}$ to $15 \mathrm{~K}$. By subtracting the spectrum at $2.1 \mathrm{~K}$ from that at $6.6 \mathrm{~K}$, we found the peak of the line was at $10045 \mathrm{~cm}^{-1}$ and the width was $610 \mathrm{~cm}^{-1}$. The integrated intensity of this line relative to the total fluorescence intensity was $\sim 0.05$.

A similar broadband emission spectrum, with a peak at $10050 \mathrm{~cm}^{-1}$ and a width of $620 \mathrm{~cm}^{-1}$, was observed in liquid $\mathrm{He}$ as shown in Fig. 5, where we also see the narrow $D_{1}$ line. No other spectral component was observed in the wave number range down to $6250 \mathrm{~cm}^{-1}$. The integrated intensity of the broad line was $\sim 0.97$ relative to the total fluorescence intensity. This line around $10000 \mathrm{~cm}^{-1}$ is assigned to be the emission from $\mathrm{Cs}^{*} \mathrm{He}_{2}$ as discussed in Sec. IV E.

\section{ANALYSIS AND DISCUSSION}

\section{A. Calculation of the emission spectra of $\mathrm{Cs}^{*} \mathrm{He}$}

We considered that the observed emission spectra in cold $\mathrm{He}$ gas were from $\mathrm{Cs}^{*} \mathrm{He}_{n}(n=1,2)$ exciplexes, and that the

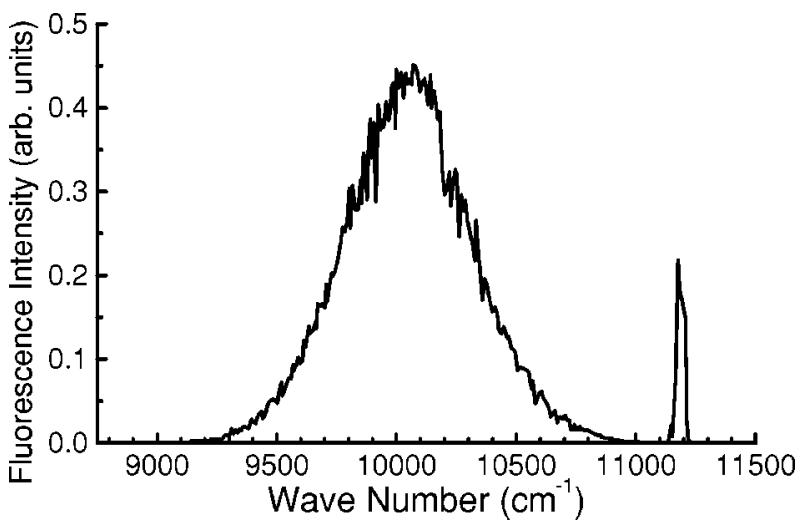

FIG. 5. Emission spectrum of Cs atoms excited through the $D_{2}$ transition $\left(12034 \mathrm{~cm}^{-1}\right)$ in superfluid liquid $\mathrm{He}$ at $1.6 \mathrm{~K}$. The broad component at about $10050 \mathrm{~cm}^{-1}$ is assigned to be the emission from $\mathrm{Cs}^{*} \mathrm{He}_{2}$ and the narrow one at $11210 \mathrm{~cm}^{-1}$ is the $D_{1}$ emission line of Cs. 
undulating structure in the case of $D_{2}$ excitation was superposition of the emission spectra from different vibrational states of $\mathrm{Cs}^{*} \mathrm{He}$. To confirm them, we calculated the emission spectra of $\mathrm{Cs}^{*} \mathrm{He}$ under the Franck-Condon approximation, using Pascale's semiempirical interatomic potentials [8]. Since these potentials are spin-orbit averaged ones, we must take account of the spin-orbit interaction, which is stronger than the interaction between $\mathrm{He}$ and $\operatorname{Cs}\left(6^{2} P\right)$ at the equilibrium internuclear distance. The method we used is the same as that in Refs. $[23,24]$. The potential curves relevant to the $\operatorname{Cs}\left(6^{2} P\right)+\operatorname{He}\left(1^{1} S_{0}\right)$ atomic system are for the $A^{2} \Pi$ and $B^{2} \Sigma$ states which are well expressed in the $\left|L S L_{z} S_{z}\right\rangle$ representation. The spin-orbit interaction is, however, diagonal in the $\left|L S J J_{z}\right\rangle$ representation. The total interaction Hamiltonian can be written in terms of the bases $\left[B^{2} \Sigma_{1 / 2}, A^{2} \Pi_{1 / 2}, A^{2} \Pi_{3 / 2}\right]$ as

$$
\left[\begin{array}{ccc}
V_{\Sigma}(R) & \frac{\sqrt{2}}{3} \Delta_{S O} & 0 \\
\frac{\sqrt{2}}{3} \Delta_{S O} & V_{\Pi}(R)-\frac{\Delta_{S O}}{3} & 0 \\
0 & 0 & V_{\Pi}(R)+\frac{\Delta_{S O}}{3}
\end{array}\right],
$$

where $\Delta_{S O}$ is the spin-orbit splitting energy of Cs in the $6{ }^{2} P$ states $\left(554.04 \mathrm{~cm}^{-1}\right), R$ is the Cs-He internuclear distance, and $V_{\Pi}(R)$ and $V_{\Sigma}(R)$ are the potential energies for the $A^{2} \Pi$ and $B^{2} \Sigma$ states, respectively. This method is based on the assumption that the spin-orbit coupling strength is independent of $R$.

Diagonalizing the matrix (1) for each $R$, we obtained the potential curves of $\mathrm{Cs}-\mathrm{He}$ as shown in Fig. 6, where the ground state potential curve is also shown. They are labeled by the dominant $\left|L S L_{z} S_{z}\right\rangle$ component at small $R$. The potential curve with the highest energy among the three excited states is that for the $B^{2} \Sigma_{1 / 2}$ state which correlates with $\operatorname{Cs}\left(6{ }^{2} P_{3 / 2}\right)+\operatorname{He}\left(1{ }^{1} S_{0}\right)$. This potential curve does not have a well deep enough to make a bound or quasibound state. The middle potential curve is for the $A^{2} \Pi_{3 / 2}$ state correlating with $\operatorname{Cs}\left(6^{2} P_{3 / 2}\right)+\operatorname{He}\left(1{ }^{1} S_{0}\right)$. This potential curve has a well at $R=3.5 \AA$ with a depth of $112 \mathrm{~cm}^{-1}$ measured from the dissociation limit and has no potential barrier. The lowest potential curve is for the $A^{2} \Pi_{1 / 2}$ state correlating with $\operatorname{Cs}\left(6{ }^{2} P_{1 / 2}\right)+\operatorname{He}\left(1{ }^{1} S_{0}\right)$. This potential has a barrier at $R$ $=5.0 \AA$ with a height of $75.2 \mathrm{~cm}^{-1}$ measured from the dissociation limit and has a well at $R=3.5 \AA$ with a depth of $52.2 \mathrm{~cm}^{-1}$ from the peak of the barrier. The potential curve for the ground state $X^{2} \Sigma_{1 / 2}$ is almost repulsive [8].

We calculated the vibrational eigenfunctions $\psi^{\prime}(R)$ and eigenenergies for the $A^{2} \Pi_{1 / 2}$ and $A^{2} \Pi_{3 / 2}$ states. The calculations were performed with Le Roy's program code LEVEL 7.4 [25], which can numerically solve the one-dimensional Schrödinger equation with the Numerov-Cooley algorithm. The $A^{2} \Pi_{1 / 2}$ state has only one quasibound vibrational state (predissociating state) and the $A^{2} \Pi_{3 / 2}$ state has five bound vibrational states. The energies of these vibrational states are listed in Table I and are also shown as dotted lines in Fig. 6.

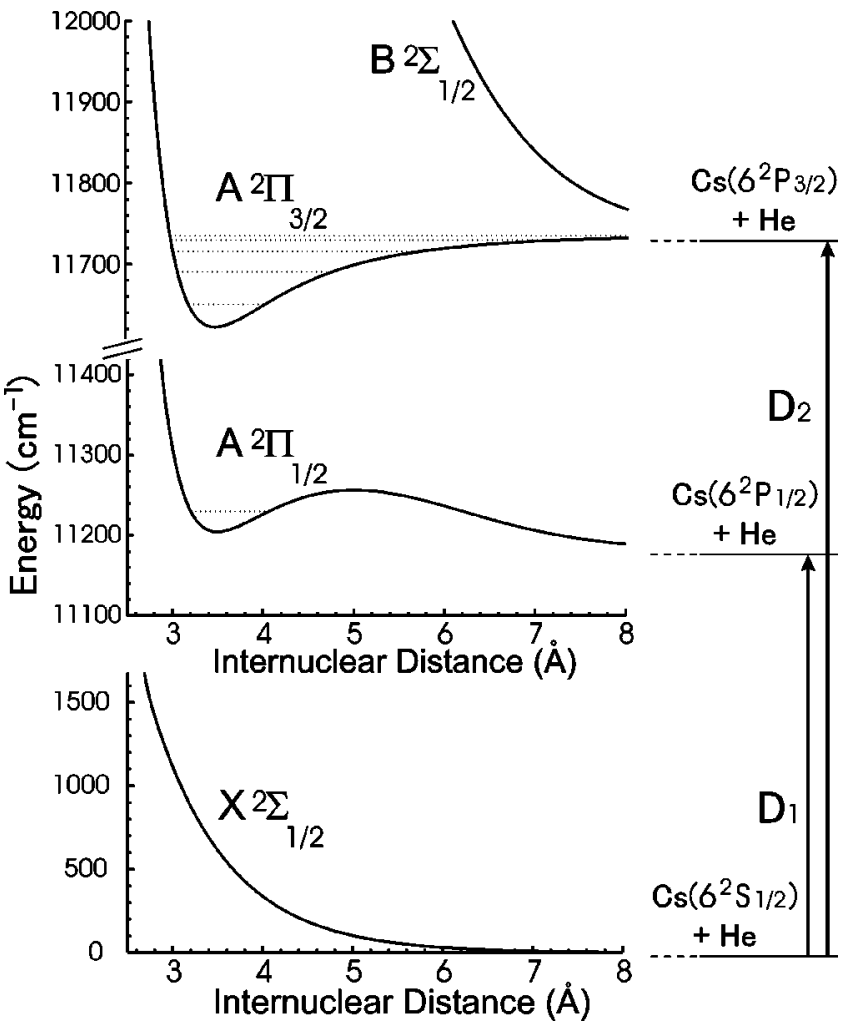

FIG. 6. Cs-He potential curves including the spin-orbit interaction (solid lines). The dotted lines show the vibrational energy levels. The electronic state of the separated atomic pair is also shown for each molecular electronic state.

The emission spectra $I(\nu)$ for the transitions from the vibrational states of these two $A^{2} \Pi$ states to the ground state $X^{2} \Sigma_{1 / 2}$ were calculated under the Franck-Condon approximation: $I(\nu) \propto \nu^{3}\left|\int d R \psi(R ; \nu) \psi^{\prime}(R)\right|^{2}$, where $\nu$ is the transition frequency, and $\psi(R ; \nu)$ is the wave function of the electronic ground state. We have neglected their rotational structure. The calculated spectra are shown in Fig. 7. We compare these theoretical spectra with the observed ones in Secs. IV B and IV C.

\section{B. Emission spectra in the case of $D_{1}$ excitation}

In the case of $D_{1}$ excitation, we observed only the $D_{1}$ emission line of $\mathrm{Cs}$ and a very weak broad spectral compo-

TABLE I. Energies of the vibrational states of $\mathrm{Cs}^{*} \mathrm{He}(v$ is the vibrational quantum number). These values are relative to the energies of $\operatorname{Cs}\left(6{ }^{2} P_{1 / 2}\right)+\operatorname{He}\left(1{ }^{1} S_{0}\right)$ and $\operatorname{Cs}\left(6{ }^{2} P_{3 / 2}\right)+\operatorname{He}\left(1{ }^{1} S_{0}\right)$ for the $A^{2} \Pi_{1 / 2}$ and $A^{2} \Pi_{3 / 2}$ states, respectively. The state with a positive energy is a quasibound state.

\begin{tabular}{cc}
\hline \hline Vibrational state & Energy $\left(\mathrm{cm}^{-1}\right)$ \\
\hline$A^{2} \Pi_{1 / 2} v=0$ & 48.35 \\
$A^{2} \Pi_{3 / 2} v=0$ & -84.89 \\
$v=1$ & -45.09 \\
$v=2$ & -19.79 \\
$v=3$ & -5.90 \\
$v=4$ & -0.51 \\
\hline
\end{tabular}






FIG. 7. Calculated emission spectra of $\mathrm{Cs}^{*} \mathrm{He}$ in the $A^{2} \Pi_{1 / 2} v$ $=0$ and $A^{2} \Pi_{3 / 2} v=0-4$ states (solid lines). The relative population of each state is normalized. The dotted lines show the Gaussian curves used in Fig. 2 (see Sec. IV C).

nent around $10695 \mathrm{~cm}^{-1}$, as seen in Fig. 3. These two components overlapped each other, so that in order to obtain the broad spectral component distinctively, we subtracted the $D_{1}$ emission line (the spectrum at $2.1 \mathrm{~K}$ ) from the spectrum observed at $30.0 \mathrm{~K}$. The resultant broad component is shown in Fig. 8. This broad component is quite similar to the theoretical spectrum for the $A^{2} \Pi_{1 / 2} v=0 \rightarrow X^{2} \Sigma_{1 / 2}$ transition, which is shown by the dotted line in Fig. 8. Thus we can conclude that this broadband emission is from $\mathrm{Cs}^{*} \mathrm{He}$ in the $A^{2} \Pi_{1 / 2} v=0$ state. The peak position of the theoretical spectrum deviates from that of the observed one by about $50 \mathrm{~cm}^{-1}$ toward larger wave number. This deviation can be attributed to errors in the potential calculation. The integrated intensity of this broadband emission was much smaller than that observed in the case of $D_{2}$ excitation. This is ascribed mainly to the existence of the potential barrier for the $A^{2} \Pi_{1 / 2}$ state.

Note that the $A^{2} \Pi_{1 / 2}$ state has only one vibrational state according to our calculation. Therefore we can clearly conclude that the observed broad spectral component can be assigned uniquely to the $A^{2} \Pi_{1 / 2} v=0 \rightarrow X^{2} \Sigma_{1 / 2}$ transition. This favorable situation is not realized for $\mathrm{Na}^{*} \mathrm{He}$ [17], $\mathrm{K}^{*} \mathrm{He}$ [17], $\mathrm{Rb} b^{*} \mathrm{He}$ [18], or even $\mathrm{Cs}^{*} \mathrm{He}$ in the $A^{2} \Pi_{3 / 2}$ state. Emission spectra of these excimers are superpositions of the components from several vibrational states, which overlap one another. Thus it is difficult to decompose it into individual components.

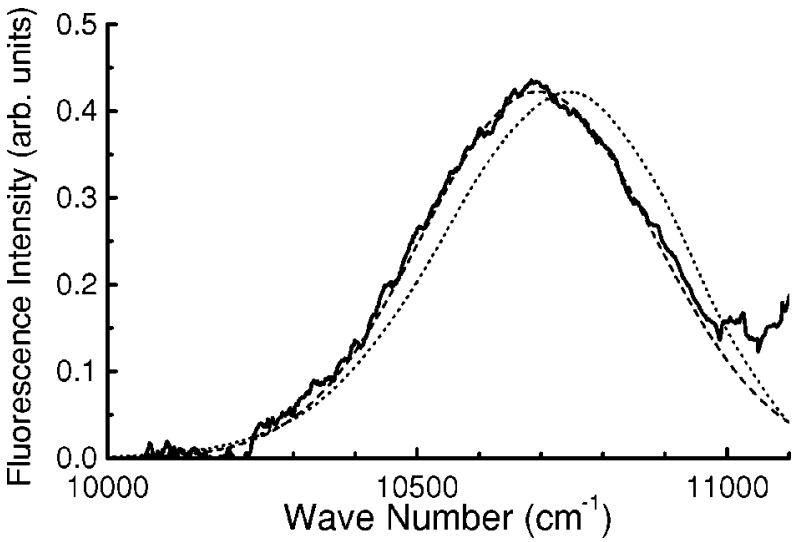

FIG. 8. Emission spectrum of $\mathrm{Cs} * \mathrm{He}$ in the $A^{2} \Pi_{1 / 2}$ state. The solid line shows the difference between the spectra observed at 30.0 $\mathrm{K}$ and $2.1 \mathrm{~K}$ in the case of $D_{1}$ excitation (see Fig. 3). The dotted line shows the theoretical spectrum. The dashed line shows the Gaussian curve with a peak at $10695 \mathrm{~cm}^{-1}$ used in Fig. 2 .

\section{Emission spectra of $\mathrm{Cs}^{*} \mathrm{He}$ in the case of $\mathrm{D}_{2}$ excitation}

When Cs atoms were excited through the $D_{2}$ line, we observed that the emission spectrum consisted of several broad spectral components. This emission spectrum is well expressed by the superposition of the six Gaussian functions shown in Fig. 2. Comparing these Gaussian curves with the theoretical spectra calculated in Sec. IV A, we found that the four broad components centered at $10695 \mathrm{~cm}^{-1}$, $11070 \mathrm{~cm}^{-1}, 11380 \mathrm{~cm}^{-1}$, and $11635 \mathrm{~cm}^{-1}$ correspond to the emission from the $A^{2} \Pi_{1 / 2} v=0, A^{2} \Pi_{3 / 2} v=0, A^{2} \Pi_{3 / 2}$ $v=1$, and $A^{2} \Pi_{3 / 2} v=2$ states of $\mathrm{Cs}^{*} \mathrm{He}$, respectively (see Fig. 7). It must be noted that there exist deviations of the peak positions between the experimental spectra and the corresponding theoretical ones. In any case, the theoretical peak is located on the high energy side of the experimental one. We believe, however, that there is no ambiguity in the above assignment, because all deviations are relatively small $\left(<125 \mathrm{~cm}^{-1}\right)$. Furthermore, the component at $10695 \mathrm{~cm}^{-1}$ has already been assigned independently from the experimental result in the case of $D_{1}$ excitation, as mentioned in Sec. IV B. The deviations probably result from the lack of precision in the pair potential curves of Cs-He. The emission spectra from the $A^{2} \Pi_{3 / 2} v \geqslant 3$ states are almost merged with the red wing of the $D_{2}$ line, and thus we cannot distinguish these components.

As described in Sec. III, we observed a slight redshift of the component at about $11070 \mathrm{~cm}^{-1}$ with decreasing $T$. We consider that this peak shift is caused by the fact that the spectrum from the $A^{2} \Pi_{3 / 2} v=0$ state almost overlaps a minor component of the spectrum from the $v=1$ state located at slightly smaller wave number (see the calculated spectra from the $v=0$ and $v=1$ states shown in Fig. 7). As mentioned in Sec. IV D, with decreasing $T$, the population of the $v=1$ state increases relative to that of the $v=0$ state, and it results in the redshift.

\section{Population distribution among vibrational states}

Here, we estimate the relative populations of vibrational states of the $A^{2} \Pi_{1 / 2}$ and $A^{2} \Pi_{3 / 2}$ states at various tempera- 


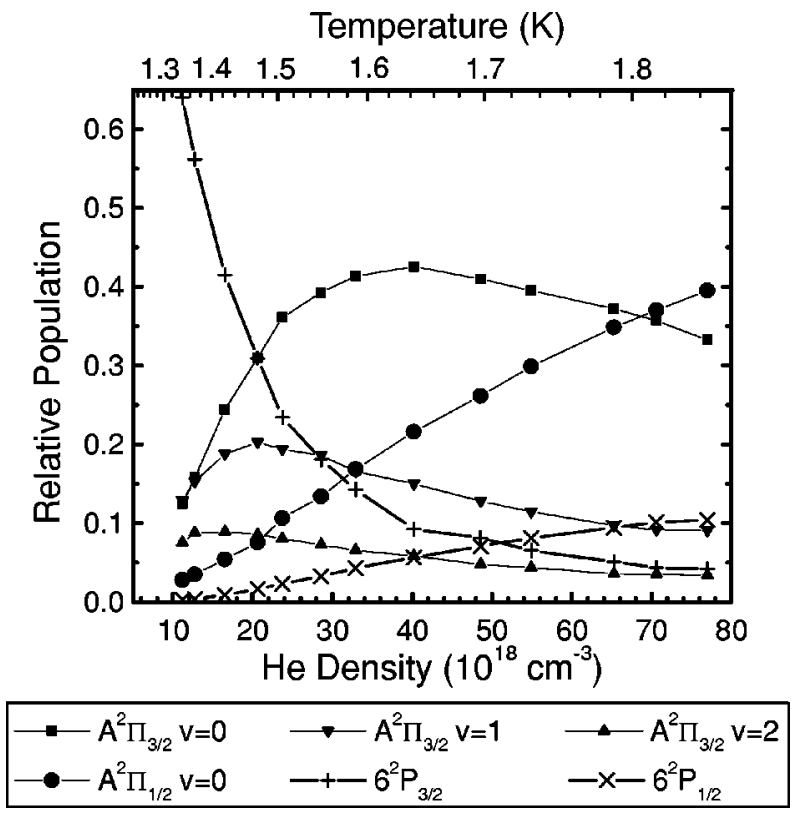

FIG. 9. He gas density dependence of the relative populations of the vibrational states of $\mathrm{Cs}{ }^{*} \mathrm{He}$ and the $6{ }^{2} P$ states of $\mathrm{Cs}$ (dots with solid lines). The cell temperature corresponding to the He gas density is shown as the upper abscissa.

tures between $1.34 \mathrm{~K}$ and $1.83 \mathrm{~K}$, in the case of $D_{2}$ excitation. We assumed that each best-fitted Gaussian curve $g_{i}(\nu)$ ( $i=1-6)$ corresponds to the strongest component of the emission spectrum from a vibrational state of $\mathrm{Cs}^{*} \mathrm{He}$ and to the Cs $D_{1}$ and $D_{2}$ lines. We estimated the relative population of each state as $\int g_{i}(\nu) \nu^{-3} d \nu$, assuming that the electronic transition moment of $\mathrm{Cs} * \mathrm{He}\left(A^{2} \Pi \rightarrow X^{2} \Sigma\right)$ is equal to that of $\mathrm{Cs}\left(6^{2} P \rightarrow 6^{2} S\right)$.

Figure 9 shows the obtained relative populations of the vibrational states of $\mathrm{Cs} * \mathrm{He}$ and the $6{ }^{2} P_{1 / 2}$ and $6{ }^{2} P_{3 / 2}$ states of $\mathrm{Cs}$ at temperatures between $1.34 \mathrm{~K}$ and $1.83 \mathrm{~K}$. In this temperature range the He gas density changes by a factor of about 7. At about $1.34 \mathrm{~K}$, where the number density of $\mathrm{He}$ atoms is about $1 \times 10^{19} \mathrm{~cm}^{-3}, D_{2}$ excitation populates mainly the $A^{2} \Pi_{3 / 2}$ state of $\mathrm{Cs}^{*} \mathrm{He}(\sim 46 \%)$ and the $6{ }^{2} P_{3 / 2}$ state of $\mathrm{Cs}(\sim 52 \%)$, and even high vibrational levels of the $A^{2} \Pi_{3 / 2}$ state are substantially populated. As the temperature goes up, the He gas density increases and the fraction of the population of $\mathrm{Cs}^{*} \mathrm{He}$ increases. Among several vibrational states of $\mathrm{Cs}^{*} \mathrm{He}$, the populations of the $A^{2} \Pi_{1 / 2} v=0$ and $A^{2} \Pi_{3 / 2} v=0$ states become dominant, because the vibrational relaxation and the electronic $A^{2} \Pi_{3 / 2} \rightarrow A^{2} \Pi_{1 / 2}$ relaxation become faster, mainly due to the increase in the number of collisions. The strong density dependence of the population distribution indicates that these relaxation times are of the same order of the radiative lifetime of $\mathrm{Cs}^{*} \mathrm{He}$ in this density range $\left(1 \times 10^{19}-8 \times 10^{19} \mathrm{~cm}^{-3}\right)$.

This density dependence gives us further interesting information concerning the predissociation from the $A^{2} \Pi_{1 / 2} v$ $=0$ state. The $\operatorname{Cs}\left(6^{2} P_{1 / 2}\right)$ atom is created by three processes; the predissociation of $\mathrm{Cs}^{*} \mathrm{He}\left(A^{2} \Pi_{1 / 2}\right)$ and the two collisional relaxations $\operatorname{Cs}\left(6{ }^{2} P_{3 / 2}\right) \rightarrow \operatorname{Cs}\left(6{ }^{2} P_{1 / 2}\right)$ and $\mathrm{Cs}{ }^{*} \mathrm{He}\left(A^{2} \Pi_{3 / 2}\right) \rightarrow \operatorname{Cs}\left(6^{2} P_{1 / 2}\right)+\operatorname{He}\left(1^{1} S_{0}\right)$. On the other hand, the $\operatorname{Cs}\left(6^{2} P_{1 / 2}\right)$ atom decays almost solely through the radiative transition. The other decay processes such as $\mathrm{Cs}\left(6{ }^{2} P_{1 / 2}\right)+\mathrm{He}\left(1^{1} S_{0}\right) \rightarrow \mathrm{Cs} * \mathrm{He}\left(A^{2} \Pi_{1 / 2}\right)$ can be neglected because the emission from $\mathrm{Cs}^{*} \mathrm{He}$ after $D_{1}$ excitation was not observed below $10 \mathrm{~K}$. In the steady state where the creation and decay rates are balanced, the predissociation rate $\gamma N_{\Pi 1 / 2}$, which is one of the creation rates, should be smaller than the spontaneous emission rate $\Gamma N_{P 1 / 2}$ (that is, $\gamma$ $\left.<\Gamma N_{P 1 / 2} / N_{\Pi 1 / 2}\right)$, where $N_{P 1 / 2}$ and $N_{\Pi 1 / 2}$ are the populations of $\operatorname{Cs}\left(6^{2} P_{1 / 2}\right)$ and $\operatorname{Cs}{ }^{*} \operatorname{He}\left(A^{2} \Pi_{1 / 2}\right)$, respectively, $\gamma$ is the rate constant for the predissociation of $\mathrm{Cs}^{*} \mathrm{He}\left(A^{2} \Pi_{1 / 2}\right)$, and $\Gamma$ is the radiative transition rate coefficient of $\operatorname{Cs}\left(6^{2} P_{1 / 2}\right)$ $\left(3.3 \times 10^{7} \mathrm{~s}^{-1}\right)$. The ratio $N_{P 1 / 2} / N_{\Pi 1 / 2}$ is less than 0.27 and is almost constant above $1.6 \mathrm{~K}$. This leads to the relation $\gamma$ $<9.0 \times 10^{6} \mathrm{~s}^{-1}$. This predissociation rate constant is much smaller than the value of $3.7 \times 10^{8} \mathrm{~s}^{-1}$, calculated theoretically with the program LEVEL 7.4 [25]. This discrepancy implies that the real potential curve for the $A^{2} \Pi_{1 / 2}$ state has a larger potential barrier and/or a lower $v=0$ level than the calculated results.

\section{E. Emission spectra of $\mathrm{Cs}^{*} \mathrm{He}_{2}$ in gaseous and liquid $\mathrm{He}$}

The broad emission line at $10045 \mathrm{~cm}^{-1}$ shown in Fig. 4 cannot be explained as fluorescence from $\mathrm{Cs}^{*} \mathrm{He}$. As in cold He gas, we observed a similar broad line in liquid $\mathrm{He}$ through $\mathrm{D}_{2}$ excitation (Fig. 5). In liquid He, the spectral components of the fluorescence from $\mathrm{Cs}^{*} \mathrm{He}$ were not observed, so that we conclude that this broad line is due to emission from $\mathrm{Cs}^{*} \mathrm{He}_{2}$. This agrees well with the fact that the energy differences of this line from the atomic $D_{1}$ and $D_{2}$ lines are approximately twice the differences from the $A^{2} \Pi_{1 / 2}$ and $A^{2} \Pi_{3 / 2} \mathrm{Cs}^{*} \mathrm{He} v=0$ states, respectively. The formation of $\mathrm{Cs}^{*} \mathrm{He}_{2}$ in liquid $\mathrm{He}$ was predicted by DupontRoc [12]. He pointed out that the valence electron of $\mathrm{Cs}\left(6^{2} P_{3 / 2}\right)$ has an applelike wave function and that two $\mathrm{He}$ atoms at maximum can attach to the $\operatorname{Cs}\left(6^{2} P_{3 / 2}\right)$ atom. This explanation agrees with our experimental result in liquid He. In $\mathrm{He}$ gas, the intensity of this broad line became maximum at temperatures $3-15 \mathrm{~K}$, but the reason for this has not been found yet.

\section{CONCLUSION}

We have observed broadband emission spectra of $\mathrm{Cs}^{*} \mathrm{He}_{n}$ $(n=1,2)$ exciplexes, by producing and exciting $\mathrm{Cs}$ atoms in cold He gas. We have assigned the spectra near the $D$ lines to be from $\mathrm{Cs}^{*} \mathrm{He}$, by calculating spectra under the FranckCondon approximation using potential curves including the spin-orbit interaction. $D_{2}$ excitation leads to strong broadband emission from the $A^{2} \Pi_{3 / 2}$ and $A^{2} \Pi_{1 / 2}$ states of Cs*He, while $D_{1}$ excitation leads to very weak emission from the $A^{2} \Pi_{1 / 2}$ state. This difference between $D_{2}$ and $D_{1}$ excitation in the intensity of the broadband emission is due to the fact that the potential curve for the $A^{2} \Pi_{1 / 2}$ state has a potential barrier and no bound states except for a quasibound state. We have also estimated the relative populations of the vibrational states of $\mathrm{Cs}^{*} \mathrm{He}$ at various temperatures below $1.9 \mathrm{~K}$, and have found that the vibrational and $A^{2} \Pi_{3 / 2}$ 
$\rightarrow A^{2} \Pi_{1 / 2}$ relaxation rates are comparable to the radiative transition rate. We have estimated the upper limit of the predissociation rate constant for $\mathrm{Cs}{ }^{*} \mathrm{He}$ in the $A^{2} \Pi_{1 / 2}$ state to be $9.0 \times 10^{6} \mathrm{~s}^{-1}$, which is two orders of magnitude smaller than the value calculated theoretically. This result as well as the deviations of the theoretical spectra from the experimental ones demand further improvement of the theoretical potential curves, especially for the excited states. The weak broad spectral component at about $10045 \mathrm{~cm}^{-1}$ is from $\mathrm{Cs}^{*} \mathrm{He}_{2}$ exciplexes. It agrees very well with the spectrum observed in liquid He. Recently, a similar investigation for $\mathrm{Rb}$ in cold He gas was carried out and the result will be reported elsewhere.

\section{ACKNOWLEDGMENTS}

We acknowledge J. Pascale for giving us the numerical Cs-He pair potential curves, and R. J. Le Roy for use of his program for calculating vibrational levels of diatomic molecules and predissociation rates. We thank also A. Hatakeyama and T. Matsuura for their contribution in the early stage of the present work. This work was supported by a Grant-in-Aid for Scientific Research of Ministry of Education, Culture, Sports, Science, and Technology of Japan (Grant No. 11304023). One of the authors (K.E.) acknowledges support from the Japan Society for the Promotion of Science.
[1] R.E.M. Hedges, D.L. Drummond, and A. Gallagher, Phys. Rev. A 6, 1519 (1972).

[2] D.L. Drummond and A. Gallagher, J. Chem. Phys. 60, 3426 (1974).

[3] G. York, R. Scheps, and A. Gallagher, J. Chem. Phys. 63, 1052 (1975).

[4] R. Scheps, Ch. Ottinger, G. York, and A. Gallagher, J. Chem. Phys. 63, 2581 (1975).

[5] M.D. Havey, S.E. Frolking, and J.J. Wright, Phys. Rev. Lett. 45, 1783 (1980).

[6] L.C. Balling, J.J. Wright, and M.D. Havey, Phys. Rev. A 26, 1426 (1982).

[7] M.V. Romalis, E. Miron, and G.D. Cates, Phys. Rev. A 56, 4569 (1997).

[8] J. Pascale, Phys. Rev. A 28, 632 (1983).

[9] As reviews, see B. Tabbert, H. Günther, and G. zu Putlitz, J. Low Temp. Phys. 109, 653 (1997); S.I. Kanorsky and A. Weis, Adv. At., Mol., Opt. Phys. 38, 87 (1997).

[10] Y. Takahashi, K. Sano, T. Kinoshita, and T. Yabuzaki, Phys. Rev. Lett. 71, 1035 (1993).

[11] S. Kanorsky, A. Weis, M. Arndt, R. Dziewior, and T.W. Hänsch, Z. Phys. B: Condens. Matter 98, 371 (1995).

[12] J. Dupont-Roc, Z. Phys. B: Condens. Matter 98, 383 (1995).

[13] T. Kinoshita, K. Fukuda, Y. Takahashi, and T. Yabuzaki, Phys. Rev. A 52, 2707 (1995).
[14] T. Kinoshita, K. Fukuda, T. Matsuura, and T. Yabuzaki, Phys. Rev. A 53, 4054 (1996).

[15] T. Eichler, R. Müller-Siebert, D. Nettels, S. Kanorsky, and A. Weis, Phys. Rev. Lett. 88, 123002 (2002).

[16] As preliminary data we observed such infrared fluorescence. For $\mathrm{Rb}$ and $\mathrm{K}$, broad emission spectra around $7000 \mathrm{~cm}^{-1}$ were observed.

[17] J. Reho, J. Higgins, C. Callegari, K.K. Lehmann, and G. Scoles, J. Chem. Phys. 113, 9686 (2000).

[18] F.R. Brühl, R.A. Trasca, and W.E. Ernst, J. Chem. Phys. 115, 10220 (2001).

[19] J. Reho, J. Higgins, K.K. Lehmann, and G. Scoles, J. Chem. Phys. 113, 9694 (2000).

[20] C.P. Schulz, P. Claas, and F. Stienkemeier, Phys. Rev. Lett. 87, 153401 (2001).

[21] Z.J. Jakubek, Q. Hui, and M. Takami, Phys. Rev. Lett. 79, 629 (1997).

[22] A. Hatakeyama, K. Enomoto, N. Sugimoto, and T. Yabuzaki, Phys. Rev. A 65, 022904 (2002).

[23] J.S. Cohen and B. Schneider, J. Chem. Phys. 61, 3230 (1974).

[24] Z.J. Jakubek and M. Takami, Chem. Phys. Lett. 265, 653 (1997).

[25] R. J. Le Roy, University of Waterloo Chemical Physics Research Report No. CP-642R 2001 (unpublished). 